Pacific

Journal of

Mathematics

\title{
THE SRANK CONJECTURE ON SCHUR'S $Q$-FUNCTIONS
}

William Y. C. Chen, Donna Q. J. Dou,

ROBERT L. TANG AND ARTHUR L. B. YANG 


\title{
THE SRANK CONJECTURE ON SCHUR'S $Q$-FUNCTIONS
}

\author{
William Y. C. Chen, Donna Q. J. Dou, \\ ROBERT L. TANG AND ARTHUR L. B. YANG
}

\begin{abstract}
We show that the shifted rank, or srank, of any partition $\lambda$ with distinct parts equals the lowest degree of the terms appearing in the expansion of Schur's $Q_{\lambda}$ function in terms of power sum symmetric functions. This gives an affirmative answer to a conjecture of Clifford. As pointed out by Clifford, the notion of the srank can be naturally extended to a skew partition $\lambda / \mu$ as the minimum number of bars among the corresponding skew bar tableaux. While the srank conjecture is not valid for skew partitions, we give an algorithm to compute the srank.
\end{abstract}

\section{Introduction}

This paper answers two open problems raised by Clifford [2005] on sranks of partitions and Schur's $Q$-functions. For any partition $\lambda$ with distinct parts, we give a proof of Clifford's srank conjecture that the lowest degree of the terms in the power sum expansion of Schur's $Q$-function $Q_{\lambda}$ is equal to the number of bars in a minimal bar tableau of shape $\lambda$. Clifford [2003; 2005] also posed the problem of determining the minimum number of bars among bar tableaux of a skew shape $\lambda / \mu$. He noted that this minimum number can be naturally regarded as the shifted rank, or srank, of $\lambda / \mu$, denoted $\operatorname{srank}(\lambda / \mu)$. For a skew bar tableau, we present an algorithm to generate a skew bar tableau without increasing the number of bars. This algorithm gives a bar tableau with the minimum number of bars.

Schur's $Q$-functions arise in the study of the projective representations of symmetric groups [Schur 1911]; see also [Morris 1962; 1979; Humphreys 1986; Nazarov 1988; Józefiak 1989; Hoffman and Humphreys 1992]. Shifted tableaux are closely related to Schur's $Q$-functions, playing a role analogous to that of ordinary tableaux for Schur functions. Sagan [1987] and Worley [1984] have independently developed a combinatorial theory of shifted tableaux, which includes shifted versions of the Robinson-Schensted-Knuth correspondence, Knuth's equivalence

MSC2000: primary 05E05; secondary 20C25.

Keywords: srank, bar tableau, Schur's $Q$-function, power sum symmetric function, strip tableau, skew bar tableau. 
relations, Schützenberger's jeu de taquin, etc. The connections between this combinatorial theory of shifted tableaux and the theory of projective representations of the symmetric groups are further explored by Stembridge [1989].

Clifford [2005] studied the srank of shifted diagrams for partitions with distinct parts. Recall that the rank of an ordinary partition is defined as the number of boxes on the main diagonal of the corresponding Young diagram. Nazarov and Tarasov [2002] found an important generalization of the rank of an ordinary partition to a skew partition in their study of tensor products of Yangian modules. A general theory of border strip decompositions and border strip tableaux of skew partitions was developed by Stanley [2002], and it has been shown that the rank of a skew partition is the least number of strips to construct a minimal border strip decomposition of the skew diagram. Motivated by Stanley's theorem, Clifford [2005] generalized the rank of a partition to the rank of a shifted partition, called srank, in terms of minimal bar tableaux.

Clifford also noticed that the srank is closely related to Schur's $Q$-function, as suggested by the work of Stanley [2002] on the rank of a partition. Stanley introduced a degree operator by taking the degree of the power sum symmetric function $p_{\mu}$ as the number of nonzero parts of the indexing partition $\mu$. Clifford and Stanley [2004] defined the bottom Schur functions to be the sum of the lowest degree terms in the expansion of the Schur functions in terms of the power sums. Clifford [2005] studied the lowest degree terms in the expansion of Schur's $Q$ functions in terms of power sum symmetric functions and conjectured that the lowest degree of Schur's $Q$-function $Q_{\lambda}$ is equal to the srank of $\lambda$. Our first result is a proof of this conjecture.

However, in general, the lowest degree of the terms, which appear in the expansion of the skew Schur's $Q$-function $Q_{\lambda / \mu}$ in terms of the power sums, is not equal to the srank of the shifted skew diagram of $\lambda / \mu$. This is different from the case for ordinary skew partitions and skew Schur functions. Instead, we will take an algorithmic approach to the computation of the srank of a skew partition. It would be interesting to find an algebraic interpretation in terms of Schur's $Q$-functions.

\section{Shifted diagrams and bar tableaux}

We adopt the notation and terminology on partitions and symmetric functions in [Macdonald 1995]. A partition $\lambda$ is a weakly decreasing sequence of positive integers $\lambda_{1} \geq \lambda_{2} \geq \cdots \geq \lambda_{k}$, denoted $\lambda=\left(\lambda_{1}, \lambda_{2}, \ldots, \lambda_{k}\right)$, and $k$ is called the length of $\lambda$, denoted $\ell(\lambda)$. For convenience we may add enough 0 's at the end of $\lambda$. If $\sum_{i=1}^{k} \lambda_{i}=n$, we say that $\lambda$ is a partition of the integer $n$, and write $\lambda \vdash n$. For each partition $\lambda$ there exists a geometric representation, known as the Young diagram, which is an array of squares in the plane justified from the top left corner 
with $\ell(\lambda)$ rows and $\lambda_{i}$ squares in the $i$-th row. A partition is said to be odd or even if it has an odd or even number of even parts. We call a partition strict if all its parts are distinct. We denote by $\mathscr{P}^{o}(n)$ the set of all partitions of $n$ with only odd parts, and by $\mathscr{D}(n)$ the set of all strict partitions of $n$. For each $\lambda \in \mathscr{D}(n)$, let $S(\lambda)$ be the shifted diagram of $\lambda$, which is obtained from the Young diagram by shifting the $i$-th row $i-1$ squares to the right for each $i>1$. For instance, here is the shifted diagram of shape $(8,7,5,3,1)$ :

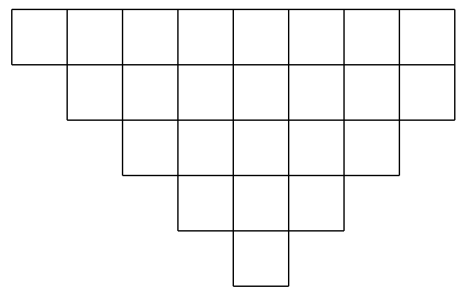

Given two partitions $\lambda$ and $\mu$ with $\lambda_{i} \geq \mu_{i}$ for each $i$, the skew partition $\lambda / \mu$ is defined to be the diagram obtained from the diagram of $\lambda$ by removing the diagram of $\mu$ at the top-left corner. Similarly, the skew shifted diagram $S(\lambda / \mu)$ is defined as the set-theoretic difference of $S(\lambda)$ and $S(\mu)$.

Now we recall the definitions of bars and bar tableaux as given in [Hoffman and Humphreys 1992]. Let $\lambda \in \mathscr{D}(n)$ be a partition with length $\ell(\lambda)=k$. Fixing an odd positive integer $r$, three subsets $I_{+}, I_{0}, I_{-}$of integers between 1 and $k$ are defined as follows:

$$
\begin{aligned}
I_{+} & =\left\{i: \lambda_{j+1}<\lambda_{i}-r<\lambda_{j} \text { for some } j \leq k\left(\text { setting } \lambda_{k+1}=0\right)\right\}, \\
I_{0} & =\left\{i: \lambda_{i}=r\right\}, \\
I_{-} & =\left\{i: r-\lambda_{i}=\lambda_{j} \text { for some } j \text { with } i<j \leq k\right\} .
\end{aligned}
$$

Set $I(\lambda, r)=I_{+} \cup I_{0} \cup I_{-}$. For each $i \in I(\lambda, r)$, we define a new strict partition $\lambda(i, r)$ of $\mathscr{D}(n-r)$ in the following way:

(1) If $i \in I_{+}$(hence $\lambda_{i}>r$ ), let $\lambda(i, r)$ be the partition obtained from $\lambda$ by removing $\lambda_{i}$ and inserting $\lambda_{i}-r$ between $\lambda_{j}$ and $\lambda_{j+1}$.

(2) If $i \in I_{0}$, let $\lambda(i, r)$ be the partition obtained from $\lambda$ by removing $\lambda_{i}$.

(3) If $i \in I_{-}$, let $\lambda(i, r)$ be the partition obtained from $\lambda$ by removing $\lambda_{i}$ and $\lambda_{j}$.

Meanwhile, for each $i \in I(\lambda, r)$, the associated $r$-bar is given as follows:

$\left(1^{\prime}\right)$ If $i \in I_{+}$, the $r$-bar consists of the rightmost $r$ squares in the $i$-th row of $S(\lambda)$, and we say that the $r$-bar is of Type 1 .

(2') If $i \in I_{0}$, the $r$-bar consists of all the squares of the $i$-th row of $S(\lambda)$, and we say that the $r$-bar is of Type 2 .

(3') If $i \in I_{-}$, the $r$-bar consists of all the squares of the $i$-th and $j$-th rows, and we say that the $r$-bar is of Type 3 . 
Thus, in the following diagram of a bar tableau of shape $(9,7,6,3,1)$, for example, the squares filled with 6 are a 7-bar of Type 1, the squares filled with 4 are a 3-bar of Type 2, and the squares filled with 3 are a 7-bar of Type 3 .

\begin{tabular}{|c|c|c|c|c|c|c|c|c|}
\hline 1 & 1 & 6 & 6 & 6 & 6 & 6 & 6 & 6 \\
\hline & 1 & 2 & 2 & 2 & 5 & 5 & 5 & \\
\hline & & 3 & 3 & 3 & 3 & 3 & 3 & \\
\hline & & & 4 & 4 & 4 & & & \\
\hline & & & & 3 & & & & \\
\hline
\end{tabular}

A bar tableau of shape $\lambda$ is an array of positive integers of shape $S(\lambda)$ subject to the following conditions:

(1) It is weakly increasing in every row.

(2) The number of parts equal to $i$ is odd for each positive integer $i$.

(3) Each positive integer $i$ can appear in at most two rows, and if $i$ appears in two rows, then these two rows must begin with $i$.

(4) For each $i$ the diagram occupied by integers less than or equal to $i$ has distinct parts.

We say that a bar tableau $T$ is of type $\rho=\left(\rho_{1}, \rho_{2}, \ldots\right)$ if the total number of $i$ 's appearing in $T$ is $\rho_{i}$. For example, the bar tableau

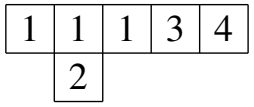

is of type $(3,1,1,1)$. The weight $\operatorname{wt}(T)$ of a bar tableau $T$ of shape $\lambda$ is defined recursively: If $T$ is empty, let $\operatorname{wt}(T)=1$. Let $\varepsilon(\lambda)$ denote the parity of the partition $\lambda$ (that is, the number of even parts of $\lambda$, modulo 2). Suppose that the largest numbers in $T$ form an $r$-bar, which is associated with an index $i \in I(\lambda, r)$. Let $j$ be the integer that occurs in the definitions of $I_{+}$and $I_{-}$. Let $T^{\prime}$ be the bar tableau of shape $\lambda(i, r)$ obtained from $T$ by removing this $r$-bar. Now, let

$$
\operatorname{wt}(T)=n_{i} \operatorname{wt}\left(T^{\prime}\right),
$$

where

$$
n_{i}= \begin{cases}(-1)^{j-i} 2^{1-\varepsilon(\lambda)}, & \text { if } i \in I_{+}, \\ (-1)^{\ell(\lambda)-i}, & \text { if } i \in I_{0}, \\ (-1)^{j-i+\lambda_{i}} 2^{1-\varepsilon(\lambda)}, & \text { if } i \in I_{-} .\end{cases}
$$

For example, the weight of the bar tableau in the figure immediately above equals

$$
(-1)^{1-1} 2^{1-0} \cdot(-1)^{1-1} 2^{1-1} \cdot(-1)^{2-2} \cdot(-1)^{1-1}=2 .
$$


The following lemma will be used in Section 3 to determine whether certain terms will vanish in the power sum expansion of Schur's $Q$-functions indexed by partitions with two distinct parts.

Lemma 2.1. Let $\lambda=\left(\lambda_{1}, \lambda_{2}\right)$ be a strict partition with two parts $\lambda_{1}$ and $\lambda_{2}$ having the same parity. Given a partition $\sigma=\left(\sigma_{1}, \sigma_{2}\right) \in \mathscr{P}^{\circ}(|\lambda|)$, if $\sigma_{2}<\lambda_{2}$, then among all bar tableaux of shape $\lambda$ there exist only two bar tableaux of type $\sigma$, say $T_{1}$ and $T_{2}$, and furthermore, we have $\mathrm{wt}\left(T_{1}\right)+\mathrm{wt}\left(T_{2}\right)=0$.

Proof. Suppose that both $\lambda_{1}$ and $\lambda_{2}$ are even. The case when $\lambda_{1}$ and $\lambda_{2}$ are odd numbers can be proved analogously. Note that $\sigma_{2}<\lambda_{2}<\lambda_{1}$. By putting 2's in the rightmost $\sigma_{2}$ squares of the second row and then filling the remaining squares in the diagram with 1's, we obtain a tableau $T_{1}$. By putting 2's in the last $\sigma_{2}$ squares of the first row and then filling the remaining squares with 1's, we obtain another tableau $T_{2}$. Clearly, both $T_{1}$ and $T_{2}$ are bar tableaux of shape $\lambda$ and type $\sigma$, and they are the only two such bar tableaux. We notice that

$$
\operatorname{wt}\left(T_{1}\right)=(-1)^{2-2} 2^{1-0} \cdot(-1)^{2-1+\lambda_{1}} 2^{1-1}=-2 .
$$

For the weight of $T_{2}$, there are two cases to consider. If $\lambda_{1}-\sigma_{2}>\lambda_{2}$, then

$$
\operatorname{wt}\left(T_{2}\right)=(-1)^{1-1} 2^{1-0} \cdot(-1)^{2-1+\lambda_{1}-\sigma_{2}} 2^{1-1}=2 .
$$

If $\lambda_{1}-\sigma_{2}<\lambda_{2}$, then

$$
\operatorname{wt}\left(T_{2}\right)=(-1)^{2-1} 2^{1-0} \cdot(-1)^{2-1+\lambda_{2}} 2^{1-1}=2 .
$$

Thus we have $\operatorname{wt}\left(T_{2}\right)=2$ in either case, so $\mathrm{wt}\left(T_{1}\right)+\mathrm{wt}\left(T_{2}\right)=0$.

For example, taking $\lambda=(8,6)$ and $\sigma=(11,3)$, the two bar tableaux $T_{1}$ and $T_{2}$ in the above lemma are like this:

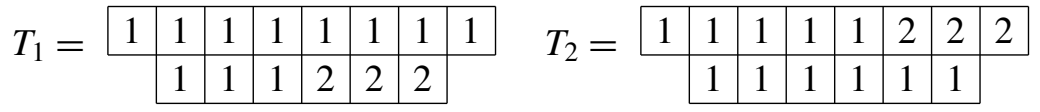

Clifford [2003] gave a natural generalization of bar tableaux to skew shapes. Formally, a skew bar tableau of shape $\lambda / \mu$ is an assignment of nonnegative integers to the squares of $S(\lambda)$ such that in addition to the four conditions (1)-(4) we impose a further one:

(5) The partition obtained by removing all squares filled with positive integers and reordering the remaining rows is $\mu$.

For example, the first diagram in Figure 1 is a skew bar tableau with shape $(8,6,5,4,1) /(8,2,1)$, and the transformations shown demonstrate this fact.

A bar tableau of shape $\lambda$ is said to be minimal if there does not exist a bar tableau with fewer bars. Motivated by the results in [Stanley 2002], Clifford [2005] defined 


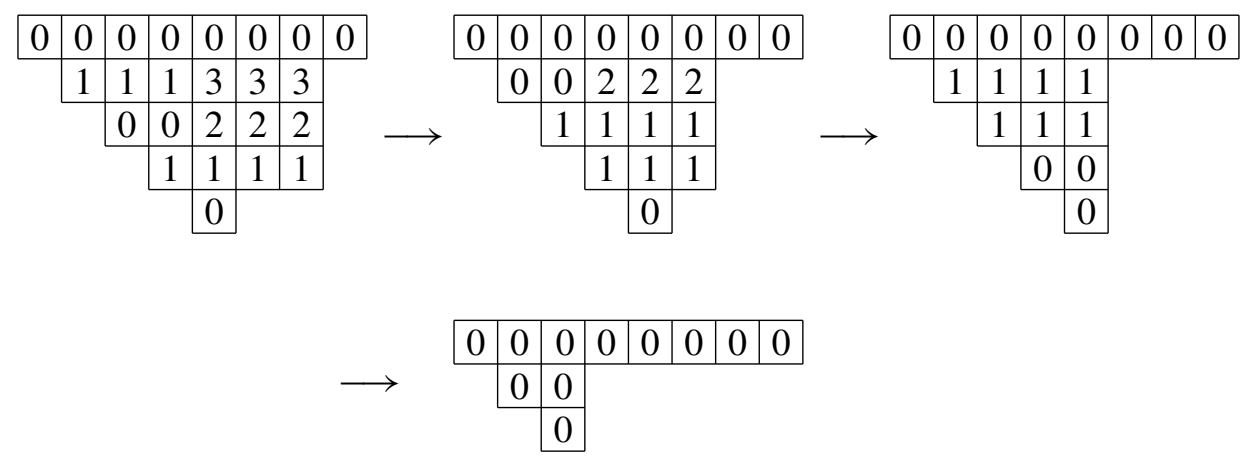

Figure 1. Checking the legality of a skew bar tableau by verifying conditions (4) and (5).

the srank of a shifted partition $S(\lambda)$, denoted $\operatorname{srank}(\lambda)$, as the number of bars in a minimal bar tableau of shape $\lambda$. Clifford also gave a formula for $\operatorname{srank}(\lambda)$ :

Theorem 2.2 [Clifford 2005, Theorem 4.1]. Given a strict partition $\lambda$, let $o$ be the number of odd parts of $\lambda$, and let $e$ be the number of nonzero even parts. Then $\operatorname{srank}(\lambda)=\max (o, e+(\ell(\lambda) \bmod 2))$.

Next we consider the number of bars in a minimal skew bar tableau of shape $\lambda / \mu$. Note that the squares filled with 0's in the skew bar tableau give rise to a shifted diagram of shape $\mu$ by reordering the rows. Let $o_{r}$ (respectively $e_{r}$ ) be the number of nonempty rows of odd (respectively even) length without 0 's, and let $o_{s}$ (respectively $e_{s}$ ) be the number of rows of $\lambda$ with some squares filled with 0 's and odd (respectively the nonzero even) number of squares not filled with 0's. For the skew bar tableau in Figure 1, we have $e_{r}=2, o_{r}=0 e_{s}=0$ and $o_{s}=1$. The following result is obvious.

Proposition 2.3. The number of bars in a minimal skew bar tableau is at least

$$
o_{s}+2 e_{s}+\max \left(o_{r}, e_{r}+\left(\left(e_{r}+o_{r}\right) \bmod 2\right)\right) .
$$

This quantity has been considered by Clifford [2003]. Observe that it depends on the positions of the 0 's.

It should be noted that a legal bar tableau of shape $\lambda / \mu$ may not exist once the positions of 0's are fixed. Similar to the case of shifted partitions, the srank of a skew shifted partition $\lambda / \mu$ is defined to be the minimal number of bars among all possible positions of 0's, denoted by $\operatorname{srank}(\lambda / \mu)$. An open problem proposed in [Clifford 2003] is to find a characterization of $\operatorname{srank}(\lambda / \mu)$. In Section 5 we will give an algorithm to compute the srank of a skew shape. 


\section{Clifford's conjecture}

In this section, we show that the lowest degree of the power sum expansion of a Schur's $Q$-function $Q_{\lambda}$ equals srank $(\lambda)$. Let us recall relevant terminology of Schur's $Q$-functions. Let $x=\left(x_{1}, x_{2}, \ldots\right)$ be an infinite sequence of independent indeterminates. We define the symmetric functions $q_{k}=q_{k}(x)$ in $x_{1}, x_{2}, \ldots$ for all integers $k$ by the following expansion of the formal power series in $t$ :

$$
\prod_{i \geq 1} \frac{1+x_{i} t}{1-x_{i} t}=\sum_{k} q_{k}(x) t^{k} .
$$

In particular, $q_{k}=0$ for $k<0$ and $q_{0}=1$. It immediately follows that

$$
\sum_{i+j=n}(-1)^{i} q_{i} q_{j}=0
$$

for all $n \geq 1$. Let $Q_{(a)}=q_{a}$ and

$$
Q_{(a, b)}=q_{a} q_{b}+2 \sum_{m=1}^{b}(-1)^{m} q_{a+m} q_{b-m} .
$$

From (3-1) we see that $Q_{(a, b)}=-Q_{(b, a)}$ and thus $Q_{(a, a)}=0$ for any $a, b$. In general, for any strict partition $\lambda$, the symmetric function $Q_{\lambda}$ is defined by the recurrence relations

$$
\begin{aligned}
Q_{\left(\lambda_{1}, \ldots, \lambda_{2 k+1}\right)} & =\sum_{m=1}^{2 k+1}(-1)^{m+1} q_{\lambda_{m}} Q_{\left(\lambda_{1}, \ldots, \widehat{\lambda}_{m}, \ldots, \lambda_{2 k+1}\right)}, \\
Q_{\left(\lambda_{1}, \ldots, \lambda_{2 k}\right)} & =\sum_{m=2}^{2 k}(-1)^{m} Q_{\left(\lambda_{1}, \lambda_{m}\right)} Q_{\left(\lambda_{2}, \ldots, \widehat{\lambda}_{m}, \ldots, \lambda_{2 k}\right)},
\end{aligned}
$$

where $\widehat{ }$ stands for a missing entry.

It was known that $Q_{\lambda}$ can be also defined as the specialization at $t=-1$ of the Hall-Littlewood functions associated with $\lambda$ [Macdonald 1995]. Originally, these $Q_{\lambda}$ symmetric functions were introduced in order to express irreducible projective characters of the symmetric groups [Schur 1911]. Note that the irreducible projective representations of $S_{n}$ are in one-to-one correspondence with partitions of $n$ with distinct parts; see [Józefiak 1989; Stembridge 1989; 1990b]. For any $\lambda \in \mathscr{D}(n)$, let $\langle\lambda\rangle$ denote the character of the irreducible projective or spin representation indexed by $\lambda$. Morris [1965] has found a combinatorial rule for calculating the characters, which is the projective analogue of the Murnaghan-Nakayama rule. In terms of bar tableaux, Morris' theorem reads as follows: 
Theorem 3.1 [Morris 1965]. Let $\lambda \in \mathscr{D}(n)$ and $\pi \in \mathscr{P}^{\circ}(n)$. Then

$$
\langle\lambda\rangle(\pi)=\sum_{T} \mathrm{wt}(T),
$$

where the sum ranges over all bar tableaux of shape $\lambda$ and type $\pi$.

This theorem for projective characters implies the following formula, which will be used later in the proof of Lemma 3.7.

Corollary 3.2. Let $\lambda$ be a strict partition of length 2. Suppose that the two parts $\lambda_{1}, \lambda_{2}$ are both odd. Then we have

$$
\langle\lambda\rangle(\lambda)=-1 .
$$

Proof. Let $T$ be the bar tableau obtained by filling the rightmost $\lambda_{2}$ squares in the first row of $S(\lambda)$ with 2's and the remaining squares with 1's, and let $T^{\prime}$ be the bar tableau obtained by filling the first row of $S(\lambda)$ with 1's and the second row with 2's. Clearly, $T$ and $T^{\prime}$ are of the same type $\lambda$. Let us first consider the weight of $T$. If $\lambda_{1}-\lambda_{2}<\lambda_{2}$, then

$$
\operatorname{wt}(T)=(-1)^{2-1} 2^{1-0} \cdot(-1)^{2-1+\lambda_{2}} 2^{1-1}=-2 .
$$

If $\lambda_{1}-\lambda_{2}>\lambda_{2}$, then

$$
\operatorname{wt}(T)=(-1)^{1-1} 2^{1-0} \cdot(-1)^{2-1+\lambda_{1}-\lambda_{2}} 2^{1-1}=-2 .
$$

In both cases, the weight of $T^{\prime}$ equals $\operatorname{wt}\left(T^{\prime}\right)=(-1)^{2-2} \cdot(-1)^{1-1}=1$. Since there are only two bar tableaux, $T$ and $T^{\prime}$, of type $\lambda$, the corollary immediately follows from Theorem 3.1.

Let $p_{k}(x)$ denote the $k$-th power sum symmetric functions, that is, $p_{k}(x)=$ $\sum_{i \geq 1} x_{i}^{k}$. For any partition $\lambda=\left(\lambda_{1}, \lambda_{2}, \ldots\right)$, let $p_{\lambda}=p_{\lambda_{1}} p_{\lambda_{2}} \cdots$. The fundamental connection between $Q_{\lambda}$ symmetric functions and the projective representations of the symmetric group is as follows.

Theorem 3.3 [Schur 1911]. Let $\lambda \in \mathscr{D}(n)$. Then we have

$$
Q_{\lambda}=\sum_{\pi \in \mathscr{P o}(n)} 2^{((\ell(\lambda)+\ell(\pi)+\varepsilon(\lambda)) / 2}\langle\lambda\rangle(\pi) \frac{p_{\pi}}{z_{\pi}},
$$

where

$$
z_{\pi}=1^{m_{1}} m_{1} ! \cdot 2^{m_{2}} m_{2} ! \cdots, \quad \text { if } \pi=\left\langle 1^{m_{1}} 2^{m_{2}} \cdots\right\rangle .
$$

Stanley [2002] introduced a degree operator on symmetric functions by defining $\operatorname{deg}\left(p_{i}\right)=1$, and $\operatorname{sodeg}\left(p_{v}\right)=\ell(v)$. Clifford [2005] applied this operator to Schur's $Q$-functions and obtained the following lower bound from Theorem 3.3.

Corollary 3.4 [Clifford 2005, Corollary 6.2]. The terms of the lowest degree in $Q_{\lambda}$ have degree at least $\operatorname{srank}(\lambda)$. 
Conjecture 3.5 [Clifford 2005, Conjecture 6.4]. The terms of the lowest degree in $Q_{\lambda}$ have degree $\operatorname{srank}(\lambda)$.

Our proof of the above conjecture depends on the Pfaffian formula for Schur's $Q$-functions. Given a skew-symmetric matrix $A=\left(a_{i j}\right)$ of even size $2 n \times 2 n$, the Pfaffian of $A$, denoted $\mathrm{Pf}(\mathrm{A})$, is defined by

$$
\operatorname{Pf}(A)=\sum_{\pi}(-1)^{\mathrm{cr}(\pi)} a_{i_{1} j_{1}} \cdots a_{i_{n} j_{n}},
$$

where the sum ranges over all set partitions $\pi$ of $\{1,2, \ldots, 2 n\}$ into two element blocks $i_{k}<j_{k}$ and $\operatorname{cr}(\pi)$ is the number of crossings of $\pi$, that is, the number of pairs $h<k$ for which $i_{h}<i_{k}<j_{h}<j_{k}$.

Theorem 3.6 [Macdonald 1995]. Given a strict partition $\lambda=\left(\lambda_{1}, \lambda_{2}, \ldots, \lambda_{2 n}\right)$ satisfying $\lambda_{1}>\cdots>\lambda_{2 n} \geq 0$, let $M_{\lambda}=\left(Q_{\left(\lambda_{i}, \lambda_{j}\right)}\right)$. Then we have

$$
Q_{\lambda}=\operatorname{Pf}\left(M_{\lambda}\right) .
$$

We first prove that Clifford's conjecture holds for strict partitions of length less than three. The proof for the general case relies on this special case.

Lemma 3.7. Let $\lambda$ be a strict partition of length $\ell(\lambda)<3$. Then the terms of the lowest degree in $Q_{\lambda}$ have degree $\operatorname{srank}(\lambda)$.

Proof. In view of Theorem 3.1 and Theorem 3.3, if there exists a unique bar tableau of shape $\lambda$ and type $\pi$, then the coefficient of $p_{\pi}$ is nonzero in the expansion of $Q_{\lambda}$. There are five cases to consider.

(1) $\ell(\lambda)=1$ and $\lambda_{1}$ is odd. Clearly, we have $\operatorname{srank}(\lambda)=1$. Note that there exists a unique bar tableau $T$ of shape $\lambda$ and of type $\lambda$ with all squares of $S(\lambda)$ filled with 1's. Therefore, the coefficient of $p_{\lambda}$ in the power sum expansion of $Q_{\lambda}$ is nonzero and the lowest degree of $Q_{\lambda}$ is 1 .

(2) $\ell(\lambda)=1$ and $\lambda_{1}$ is even. We see that $\operatorname{srank}(\lambda)=2$. Since the bars are all of odd size, there does not exist any bar tableau of shape $\lambda$ and of type $\lambda$. But there is a unique bar tableau $T$ of shape $\lambda$ and of type $\left(\lambda_{1}-1,1\right)$, which is obtained by filling the rightmost square of $S(\lambda)$ with 2 and the remaining squares with 1's. So the coefficient of $p_{\left(\lambda_{1}-1,1\right)}$ in the power sum expansion of $Q_{\lambda}$ is nonzero and the terms of the lowest degree in $Q_{\lambda}$ have degree 2.

(3) $\ell(\lambda)=2$ and the two parts $\lambda_{1}, \lambda_{2}$ have different parity. In this case, we have $\operatorname{srank}(\lambda)=1$. Note that there exists a unique bar tableau $T$ of shape $\lambda$ and of type $\left(\lambda_{1}+\lambda_{2}\right)$, which is obtained by filling all the squares of $S(\lambda)$ with 1's. Thus, the coefficient of $p_{\lambda_{1}+\lambda_{2}}$ in the power sum expansion of $Q_{\lambda}$ is nonzero and the terms of lowest degree in $Q_{\lambda}$ have degree 1 . 
(4) $\ell(\lambda)=2$ and the two parts $\lambda_{1}, \lambda_{2}$ are both even. It is easy to see $\operatorname{srank}(\lambda)=2$. Since there exists a unique bar tableau $T$ of shape $\lambda$ and of type $\left(\lambda_{1}-1, \lambda_{2}+1\right)$, which is obtained by filling the rightmost $\lambda_{2}+1$ squares in the first row of $S(\lambda)$ with 2's and the remaining squares with 1's, the coefficient of $p_{\left(\lambda_{1}-1, \lambda_{2}+1\right)}$ in the power sum expansion of $Q_{\lambda}$ is nonzero; hence the lowest degree of $Q_{\lambda}$ is equal to 2 .

(5) $\ell(\lambda)=2$ and the two parts $\lambda_{1}, \lambda_{2}$ are both odd. In this case, $\operatorname{srank}(\lambda)=2$. By Corollary 3.2, the coefficient of $p_{\lambda}$ in the power sum expansion of $Q_{\lambda}$ is nonzero, and therefore the terms of the lowest degree in $Q_{\lambda}$ have degree 2.

Given a strict partition $\lambda$, we consider the Pfaffian expansion of $Q_{\lambda}$ as shown in Theorem 3.6. To prove Clifford's conjecture, we need to determine which terms may appear in the expansion of $Q_{\lambda}$ in terms of power sum symmetric functions. Suppose that the Pfaffian expansion of $Q_{\lambda}$ is as follows:

$$
\operatorname{Pf}\left(M_{\lambda}\right)=\sum_{\pi}(-1)^{\operatorname{cr}(\pi)} Q_{\left(\lambda_{\pi_{1}}, \lambda_{\pi_{2}}\right)} \cdots Q_{\left(\lambda_{\pi_{2 m-1}}, \lambda_{\pi_{2 m}}\right)},
$$

where the sum ranges over all set partitions $\pi$ of $\{1,2, \ldots, 2 m\}$ into two element blocks $\left\{\left(\pi_{1}, \pi_{2}\right), \ldots,\left(\pi_{2 m-1}, \pi_{2 m}\right)\right\}$ with $\pi_{1}<\pi_{3}<\cdots<\pi_{2 m-1}$ and $\pi_{2 k-1}<\pi_{2 k}$ for any $k$. For the above expansion of $Q_{\lambda}$, the following two lemmas will be used to choose certain lowest degree terms in the power sum expansion of $Q_{\left(\lambda_{i}, \lambda_{j}\right)}$ in the matrix $M_{\lambda}$.

Lemma 3.8. Suppose that $\lambda$ has both odd parts and nonzero even parts. Let $\lambda_{i_{1}}$ (respectively $\lambda_{j_{1}}$ ) be the largest odd (respectively even) part of $\lambda$. If the power sum symmetric function $p_{\lambda_{i_{1}}+\lambda_{j_{1}}}$ appears in the terms of lowest degree originated from the product $Q_{\left(\lambda_{\pi_{1}}, \lambda_{\pi_{2}}\right)} \cdots Q_{\left(\lambda_{\pi_{2 m-1}}, \lambda_{\pi_{2 m}}\right)}$ as in the expansion (3-7), then we have $\left\{\pi_{1}, \pi_{2}\right\}=\left\{i_{1}, j_{1}\right\}$.

Proof. Without loss of generality, we may assume that $\lambda_{i_{1}}>\lambda_{j_{1}}$. By Lemma 3.7, the term $p_{\lambda_{i_{1}}+\lambda_{j_{1}}}$ appears in $Q_{\left(\lambda_{i_{1}}, \lambda_{j_{1}}\right)}$ with a nonzero coefficient. Since $\lambda_{i_{1}}, \lambda_{j_{1}}$ are the largest odd and even parts, $p_{\lambda_{i_{1}}+\lambda_{j_{1}}}$ does not appear as a factor of any term of the lowest degree in the expansion of any other $Q_{\left(\lambda_{i_{k}}, \lambda_{j_{k}}\right)}$, where $\lambda_{i_{k}}$ and $\lambda_{j_{k}}$ have different parity. Meanwhile, if $\lambda_{i_{k}}$ and $\lambda_{j_{k}}$ have the same parity, then we consider the bar tableaux of shape $\left(\lambda_{i_{k}}, \lambda_{j_{k}}\right)$ and of type $\left(\lambda_{i_{1}}+\lambda_{j_{1}}, \lambda_{i_{k}}+\lambda_{j_{k}}-\lambda_{i_{1}}-\lambda_{j_{1}}\right)$. Observe that $\lambda_{i_{k}}+\lambda_{j_{k}}-\lambda_{i_{1}}-\lambda_{j_{1}}<\lambda_{j_{k}}$. Since the lowest degree of $Q_{\left(\lambda_{i_{k}}, \lambda_{j_{k}}\right)}$ is 2 , from Lemma 2.1 it follows that $p_{\lambda_{i_{1}}+\lambda_{j_{1}}}$ can not be a factor of any term of lowest degree in the power sum expansion of $Q_{\left(\lambda_{i_{k}}, \lambda_{j_{k}}\right)}$.

Lemma 3.9. Suppose that $\lambda$ only has even parts. Let $\lambda_{1}, \lambda_{2}$ be the two largest parts of $\lambda$ (allowing $\lambda_{2}=0$ ). If the power sums $p_{\lambda_{1}-1} p_{\lambda_{2}+1}$ appears in the terms of the lowest degree given by the product $Q_{\left(\lambda_{\pi_{1}}, \lambda_{\pi_{2}}\right)} \cdots Q_{\left(\lambda_{\pi_{2 m-1}}, \lambda_{\pi_{2 m}}\right)}$ as in (3-7), then we have $\left(\pi_{1}, \pi_{2}\right)=(1,2)$. 
Proof. From Case (4) of the proof of Lemma 3.7 it follows that $p_{\lambda_{1}-1} p_{\lambda_{2}+1}$ appears as a term of the lowest degree in the power sum expansion of $Q_{\left(\lambda_{1}, \lambda_{2}\right)}$. We next consider the power sum expansion of any other $Q_{\left(\lambda_{i}, \lambda_{j}\right)}$. First, we consider the case when $\lambda_{i}+\lambda_{j}>\lambda_{2}+1$ and $\lambda_{i} \leq \lambda_{2}$. Since $\lambda_{i}+\lambda_{j}-\left(\lambda_{2}+1\right)<\lambda_{j}$, by Lemma 2.1 , the term $p_{\lambda_{2}+1}$ is not a factor of any term of the lowest degree in the power sum expansion of $Q_{\left(\lambda_{i}, \lambda_{j}\right)}$. Now we are left with the case when $\lambda_{i}+\lambda_{j}>\lambda_{1}-1$ and $\lambda_{i} \leq \lambda_{1}-2$. Since $\lambda_{i}+\lambda_{j}-\left(\lambda_{1}-1\right)<\lambda_{j}$, by Lemma 2.1 the term $p_{\lambda_{1}-1}$ does not appear as a factor in the terms of the lowest degree of $Q_{\left(\lambda_{i}, \lambda_{j}\right)}$. So we have shown that if either $p_{\lambda_{2}+1}$ or $p_{\lambda_{1}-1}$ appears as a factor of some lowest degree term for $Q_{\left(\lambda_{i}, \lambda_{j}\right)}$, then we deduce that $\lambda_{i}=\lambda_{1}$. Moreover, if both $p_{\lambda_{1}-1}$ and $p_{\lambda_{2}+1}$ are factors of the lowest degree terms in the power sum expansion of $Q_{\left(\lambda_{1}, \lambda_{j}\right)}$, then we have $\lambda_{j}=\lambda_{2}$.

We now present the main result of this paper.

Theorem 3.10. For any $\lambda \in \mathscr{D}(n)$, the terms of the lowest degree in $Q_{\lambda}$ have degree $\operatorname{srank}(\lambda)$.

Proof. We write the strict partition $\lambda$ in the form $\left(\lambda_{1}, \lambda_{2}, \ldots, \lambda_{2 m}\right)$, where the $\lambda_{i}$ 's satisfy $\lambda_{1}>\cdots>\lambda_{2 m} \geq 0$. Suppose that the partition $\lambda$ has $o$ odd parts and $e$ nonzero even parts. For the sake of presentation, let $\left(\lambda_{i_{1}}, \lambda_{i_{2}}, \ldots, \lambda_{i_{o}}\right)$ denote the sequence of odd parts in decreasing order, and let $\left(\lambda_{j_{1}}, \lambda_{j_{2}}, \ldots, \lambda_{j_{e}}\right)$ denote the sequence of nonzero even parts in decreasing order.

We first consider the case $o \geq e$. In this case, it will be shown that $\operatorname{srank}(\lambda)=o$. By Theorem 2.2, if $\lambda_{2 m}>0$, that is, $\ell(\lambda)=2 m$, we have $\operatorname{srank}(\lambda)=\max (o, e+0)=$ $o$. If $\lambda_{2 m}=0$, that is, $\ell(\lambda)=2 m-1$, we still have $\operatorname{srank}(\lambda)=\max (o, e+1)=o$.

Let

$$
A=p_{\lambda_{i_{1}}+\lambda_{j_{1}}} \cdots p_{\lambda_{i_{e}}+\lambda_{j_{e}}} p_{\lambda_{i_{e+1}}} p_{\lambda_{i_{e+2}}} \cdots p_{\lambda_{i_{o}}} .
$$

We claim that $A$ appears as a term of the lowest degree in the power sum expansion of $Q_{\lambda}$. To this end, we need to determine those matchings $\pi$ of $\{1,2, \ldots, 2 m\}$ in (3-7) for which the power sum expansion of the product $Q_{\left(\lambda_{\pi_{1}}, \lambda_{\pi_{2}}\right)} \cdots Q_{\left(\lambda_{\pi_{2 m-1}}, \lambda_{\pi_{2 m}}\right)}$ contains $A$ as a term of the lowest degree.

By Lemma 3.8, if the $p_{\lambda_{i_{1}}+\lambda_{j_{1}}}$ appears as a factor in the lowest degree terms of

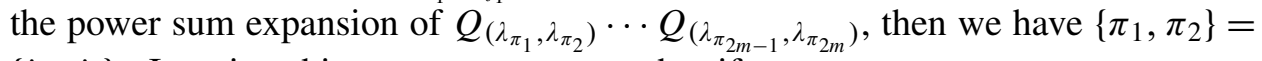
$\left\{i_{1}, j_{1}\right\}$. Iterating this argument, we see that if $p_{\lambda_{i_{1}}+\lambda_{j_{1}}} \cdots p_{\lambda_{i_{e}}+\lambda_{j_{e}}}$ appears as a factor in the lowest degree terms of $Q_{\left(\lambda_{\pi_{1}}, \lambda_{\pi_{2}}\right)} \cdots Q_{\left(\lambda_{\pi_{2 m-1}}, \lambda_{\pi_{2 m}}\right)}$, then we have

$$
\left\{\pi_{1}, \pi_{2}\right\}=\left\{i_{1}, j_{1}\right\}, \ldots,\left\{\pi_{2 e-1}, \pi_{2 e}\right\}=\left\{i_{e}, j_{e}\right\} .
$$

It remains to determine the ordered pairs $\left\{\left(\pi_{2 e+1}, \pi_{2 e+2}\right), \ldots,\left(\pi_{2 m-1}, \pi_{2 m}\right)\right\}$. By the argument of Case (5) of the proof of Lemma 3.7, for any $e+1 \leq k<l \leq o$, the term $p_{\lambda_{i_{k}}} p_{\lambda_{i_{l}}}$ appears as a term of the lowest degree in the power sum expansion of $Q_{\left(\lambda_{i_{k}}, \lambda_{i_{l}}\right)}$. Also, if the power sum symmetric function $p_{\lambda_{i_{e+1}}} p_{\lambda_{i_{e+2}}} \cdots p_{\lambda_{i_{o}}}$ 
appears as a term of the lowest degree in the power sum expansion of the product

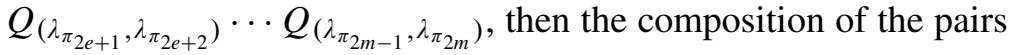

$$
\left\{\left(\pi_{2 e+1}, \pi_{2 e+2}\right), \ldots,\left(\pi_{2 m-1}, \pi_{2 m}\right)\right\}
$$

could be any matching of $\{1,2, \ldots, 2 m\} \backslash\left\{i_{1}, j_{1}, \ldots, i_{e}, j_{e}\right\}$.

To summarize, there are $(2(m-e)-1) ! !$ matchings $\pi$ where $A$ appears as a term of the lowest degree in the power sum expansion of $Q_{\left(\lambda_{\pi_{1}}, \lambda_{\pi_{2}}\right)} \cdots Q_{\left(\lambda_{\pi_{2 m-1}}, \lambda_{\pi_{2 m}}\right)}$. Combining Corollary 3.2 and Theorem 3.3, we find that the coefficient of $p_{\lambda_{i_{k}}} p_{\lambda_{i_{l}}}$ $(e+1 \leq k<l \leq o)$ in the power sum expansion of $Q_{\left(\lambda_{i_{k}}, \lambda_{i_{l}}\right)}$ is $-4 /\left(\lambda_{i_{k}} \lambda_{i_{l}}\right)$, while the coefficient of $p_{\lambda_{i_{k}}}(e+1 \leq k \leq o)$ in the power sum expansion of $Q_{\left(\lambda_{i_{k}}, 0\right)}$ is $2 / \lambda_{i_{k}}$. Thus the coefficient of $A$ in the expansion of the product $Q_{\left(\lambda_{\pi_{1}}, \lambda_{\pi_{2}}\right)} \cdots Q_{\left(\lambda_{\pi_{2 m-1}}, \lambda_{\pi_{2 m}}\right)}$ is independent of the choice of $\pi$. Since $(2(m-e)-1)$ !! is an odd number, the term $A$ will not vanish in the expansion of $Q_{\lambda}$. Note that the degree of $A$ is $e+(o-e)=o$, which is equal to $\operatorname{srank}(\lambda)$, as desired.

Similarly, we consider the case $e>o$. Set $e^{\prime}=e+(\ell(\lambda) \bmod 2)$, and let $\left(\lambda_{j_{1}}, \lambda_{j_{2}}, \ldots, \lambda_{j_{e^{\prime}}}\right)$ be the sequence of even parts in decreasing order. By Theorem 2.2, we have $\operatorname{srank}(\lambda)=\max \left(o, e^{\prime}\right)=e^{\prime}$. Let

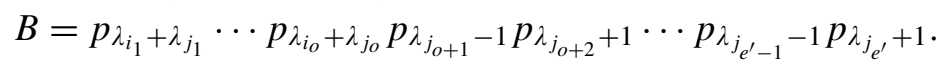

We proceed to prove that $B$ appears as a term of the lowest degree in the power sum expansion of $Q_{\lambda}$. Applying Lemma 3.8 repeatedly, we deduce that if the term $p_{\lambda_{i_{1}}+\lambda_{j_{1}}} \cdots p_{\lambda_{i_{o}}+\lambda_{j_{o}}}$ appears as a factor in the lowest degree terms of the product $Q_{\left(\lambda_{\pi_{1}}, \lambda_{\pi_{2}}\right)} \cdots Q_{\left(\lambda_{\pi_{2 m-1}}, \lambda_{\pi_{2 m}}\right)}$, then

$$
\left\{\pi_{1}, \pi_{2}\right\}=\left\{i_{1}, j_{1}\right\}, \ldots,\left\{\pi_{2 o-1}, \pi_{2 o}\right\}=\left\{i_{o}, j_{o}\right\} .
$$

On the other hand, iteration of Lemma 3.9 reveals that if the power sum symmetric function $p_{\lambda_{j_{o+1}}-1} p_{\lambda_{j_{o+2}}+1} \cdots p_{\lambda_{j_{e^{\prime}-1}-1}} p_{\lambda_{j_{e^{\prime}}+1}}$ appears as a term of the lowest degree in the power sum expansion of $Q_{\left(\lambda_{\pi_{2 o+1}}, \lambda_{\pi_{2 o+2}}\right)} \cdots Q_{\left(\lambda_{\pi_{2 m-1}}, \lambda_{\pi_{2 m}}\right)}$, then

$$
\left\{\pi_{2 o+1}, \pi_{2 o+2}\right\}=\left\{j_{o+1}, j_{o+2}\right\}, \ldots,\left\{\pi_{2 m-1}, \pi_{2 m}\right\}=\left\{j_{e^{\prime}-1}, j_{e^{\prime}}\right\} .
$$

Therefore, if $B$ appears as a term of the lowest degree in the power sum expansion

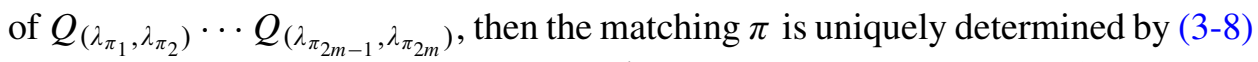
and (3-9). Note that the degree of $B$ is $e^{\prime}$, which coincides with $\operatorname{srank}(\lambda)$.

Since there is always a term of $\operatorname{degree} \operatorname{srank}(\lambda)$ in the power sum expansion of $Q_{\lambda}$, the theorem follows.

\section{Skew Schur's $Q$-functions}

In this section, we show that $\operatorname{srank}(\lambda / \mu)$ is a lower bound of the lowest degree of the terms in the power sum expansion of the skew Schur's $Q$-function $Q_{\lambda / \mu}$. Note that Clifford's conjecture does not hold for skew shapes. 
We first recall a definition of the skew Schur's $Q$-function in terms of strip tableaux. The concept of strip tableaux was introduced by Stembridge [1990b] to describe the Morris rule for the evaluation of irreducible spin characters. Given a skew partition $\lambda / \mu$, the $j$-th diagonal of the skew shifted diagram $S(\lambda / \mu)$ is defined as the set of squares $(1, j),(2, j+1),(3, j+2), \ldots$ in $S(\lambda / \mu)$. A skew diagram $S(\lambda / \mu)$ is called a strip if it is connected and each diagonal contains at most one box. The height $h$ of a strip is defined to be the number of rows it occupies. A double strip is a skew diagram formed by the union of two strips which both start on the diagonal consisting of squares $(j, j)$. The depth of a double strip is defined to be $\alpha+\beta$ if it has $\alpha$ diagonals of length two and its diagonals of length one occupy $\beta$ rows. A strip tableau of shape $\lambda / \mu$ and type $\pi=\left(\pi_{1}, \ldots, \pi_{k}\right)$ is defined to be a sequence of shifted diagrams

$$
S(\mu)=S\left(\lambda^{0}\right) \subseteq S\left(\lambda^{1}\right) \subseteq \cdots \subseteq S\left(\lambda^{k}\right)=S(\lambda)
$$

with $\left|\lambda^{i} / \lambda^{i-1}\right|=\pi_{i}(1 \leq i \leq k)$ such that each skew shifted diagram $S\left(\lambda^{i} / \lambda^{i-1}\right)$ is either a strip or a double strip.

The skew Schur's $Q$-function can be defined as the weight generating function of strip tableaux in the following way. For a strip of height $h$ we assign the weight $(-1)^{h-1}$, and for a double strip of depth $d$ we assign the weight $2(-1)^{d-1}$. The weight of a strip tableau $T$, denoted $\operatorname{wt}(T)$, is the product of the weights of strips and double strips of which $T$ is composed. Then the skew Schur's $Q$-function $Q_{\lambda / \mu}$ is given by

$$
Q_{\lambda / \mu}=\sum_{\pi \in \mathscr{P}^{\circ}(|\lambda / \mu|)} \sum_{T} 2^{\ell(\pi)} \operatorname{wt}(T) \frac{p_{\pi}}{z_{\pi}},
$$

where $T$ ranges over all strip tableaux $T$ of shape $\lambda / \mu$ and type $\pi$; see [Stembridge 1990b, Theorem 5.1].

We have the following Pfaffian formula for the skew Schur's $Q$-function.

Theorem 4.1 [Józefiak and Pragacz 1991]. Let $\lambda$ and $\mu$ be strict partitions with $m=\ell(\lambda), n=\ell(\mu), \mu \subset \lambda$, and let $M(\lambda, \mu)$ denote the skew-symmetric matrix

$$
\left(\begin{array}{cc}
A & B \\
-B^{t} & 0
\end{array}\right)
$$

where $A=\left(Q_{\left(\lambda_{i}, \lambda_{j}\right)}\right)$ and $B=\left(Q_{\left(\lambda_{i}-\mu_{n+1-j}\right)}\right)$.

(1) If $m+n$ is even, we have $Q_{\lambda / \mu}=\operatorname{Pf}(M(\lambda, \mu))$.

(2) If $m+n$ is odd, we have $Q_{\lambda / \mu}=\operatorname{Pf}\left(M\left(\lambda, \mu^{\prime}\right)\right)$, where $\mu^{\prime}=\left(\mu_{1}, \ldots, \mu_{n}, 0\right)$.

A combinatorial proof of the above theorem was given by Stembridge [1990a] in terms of lattice paths, and later, Hamel [1996] gave an interesting generalization by using the border strip decompositions of the shifted diagram. 
Given a skew partition $\lambda / \mu$, Clifford [2003] constructed a bijection between skew bar tableaux of shape $\lambda / \mu$ and skew strip tableaux of the same shape, which preserves the type of the tableau. Using this bijection, it is straightforward to derive the following result.

Proposition 4.2. The terms of the lowest degree in $Q_{\lambda / \mu}$ have degree at least $\operatorname{srank}(\lambda / \mu)$.

Different from the case of nonskew shapes, in general, the lowest degree terms in $Q_{\lambda / \mu}$ do not have the degree $\operatorname{srank}(\lambda / \mu)$. For example, take the skew partition $(4,3) /(3)$. It is easy to see that $\operatorname{srank}((4,3) /(3))=2$. While, using Theorem 4.1 and Stembridge's SF package for Maple [Stembridge], we obtain that

$$
Q_{(4,3) /(3)}=\operatorname{Pf}\left(\begin{array}{cccc}
0 & Q_{(4,3)} & Q_{(4)} & Q_{(1)} \\
Q_{(3,4)} & 0 & Q_{(3)} & Q_{(0)} \\
-Q_{(4)} & -Q_{(3)} & 0 & 0 \\
-Q_{(1)} & -Q_{(0)} & 0 & 0
\end{array}\right)=2 p_{1}^{4} .
$$

This shows that the lowest degree of $Q_{(4,3) /(3)}$ equals 4 , which is strictly greater than $\operatorname{srank}((4,3) /(3))$.

\section{The srank of skew partitions}

In this section, we present an algorithm to determine the srank for the skew partition $\lambda / \mu$. In fact, the algorithm leads to a configuration of 0 's. To obtain the srank of a skew partition, we need to minimize the number of bars by adjusting the positions of 0's. Given a configuration $\mathscr{b}$ of 0 's in the shifted diagram $S(\lambda)$, let

$$
\kappa(\mathscr{C})=o_{s}+2 e_{s}+\max \left(o_{r}, e_{r}+\left(\left(e_{r}+o_{r}\right) \bmod 2\right)\right),
$$

where $o_{r}$ (respectively $e_{r}$ ) counts the number of nonempty rows in which there are an odd (respectively even) number of squares and no squares are filled with 0 , and $o_{s}$ (respectively $e_{s}$ ) records the number of rows in which at least one square is filled with 0 but there are an odd (respectively nonzero even) number of squares not labeled 0 .

If there exists at least one bar tableau of shape $\lambda / \mu$ under some configuration $\mathscr{C}$, we say that $\mathscr{C}$ is admissible. For a fixed configuration $\mathscr{C}$, each row is one of the following eight possible types:

(1) an even row bounded by an even number of 0's, denoted $(e, e)$,

(2) an odd row bounded by an even number of 0 's, denoted $(e, o)$,

(3) an odd row bounded by an odd number of 0's, denoted $(o, e)$,

(4) an even row bounded by an odd number of 0 's, denoted $(o, o)$, 
(5) an even row without 0 's, denoted $(\varnothing, e)$,

(6) an odd row without 0 's, denoted $(\varnothing, o)$,

(7) an even row filled with 0 's, denoted $(e, \varnothing)$,

(8) an odd row filled with 0 's, denoted $(o, \varnothing)$.

Given two rows with respective types $s$ and $s^{\prime}$ for some configuration $\mathscr{C}$, if we can obtain a new configuration $\mathscr{C}^{\prime}$ by exchanging the locations of 0 's in these two rows such that their new types are $t$ and $t^{\prime}$ respectively, then denote it by

$$
\mathscr{C}^{\prime}=\mathscr{C}\left(\left[\begin{array}{l}
s \\
s^{\prime}
\end{array}\right] \rightarrow\left[\begin{array}{l}
t \\
t^{\prime}
\end{array}\right]\right) .
$$

Let $o_{r}, e_{r}, o_{s}, e_{s}$ be defined as above corresponding to configuration $\mathscr{C}$, and let $o_{r}^{\prime}$, $e_{r}^{\prime}, o_{s}^{\prime}, e_{s}^{\prime}$ be those of $\mathscr{C}^{\prime}$.

We will show how the quantity $\kappa(\mathscr{C})$ changes when exchanging the locations of 0 's in $\mathscr{C}$.

\section{Lemma 5.1. If}

$$
\mathscr{C}^{\prime}=\mathscr{C}\left(\left[\begin{array}{l}
s \\
s^{\prime}
\end{array}\right] \rightarrow\left[\begin{array}{l}
s \\
s^{\prime}
\end{array}\right]\right) \quad \text { or } \quad \mathscr{C}^{\prime}=\mathscr{C}\left(\left[\begin{array}{l}
s \\
s^{\prime}
\end{array}\right] \rightarrow\left[\begin{array}{l}
s^{\prime} \\
s
\end{array}\right]\right),
$$

that is, the types of the two involved rows are remained or exchanged, where $s, s^{\prime}$ are any two possible types, then $\kappa\left(\mathscr{C}^{\prime}\right)=\kappa(\mathscr{C})$.

Lemma 5.2. If $\mathscr{C}^{\prime}=\mathscr{C}\left(\left[\begin{array}{c}(e, e) \\ (\varnothing, o)\end{array}\right] \rightarrow\left[\begin{array}{c}(\varnothing, e) \\ (e, o)\end{array}\right]\right)$, then $\kappa\left(\mathscr{C}^{\prime}\right) \leq \kappa(\mathscr{C})$.

Proof. In this case we have

$$
o_{s}^{\prime}=o_{s}+1, \quad e_{s}^{\prime}=e_{s}-1, \quad o_{r}^{\prime}=o_{r}-1, \quad e_{r}^{\prime}=e_{r}+1 .
$$

Note that $o_{r}+e_{r}=\ell(\lambda)-\ell(\mu)$. Now there are two cases to consider.

Case I. The skew partition $\lambda / \mu$ satisfies that $\ell(\lambda)-\ell(\mu) \equiv 0(\bmod 2)$.

(1) If $o_{r} \leq e_{r}$, then $o_{r}^{\prime} \leq e_{r}^{\prime}$ and

$\kappa(\mathscr{C})=o_{s}+2 e_{s}+e_{r}, \quad \kappa\left(\mathscr{C}^{\prime}\right)=o_{s}+1+2\left(e_{s}-1\right)+e_{r}^{\prime}=o_{s}+2 e_{s}+e_{r}=\kappa(\mathscr{C})$.

(2) If $o_{r} \geq e_{r}+2$, then $o_{r}^{\prime}=o_{r}-1 \geq e_{r}+1=e_{r}^{\prime}$ and

$\kappa(\mathscr{C})=o_{s}+2 e_{s}+o_{r}, \quad \kappa\left(\mathscr{C}^{\prime}\right)=o_{s}+2 e_{s}-1+o_{r}^{\prime}=o_{s}+2 e_{s}+o_{r}-2<\kappa(\mathscr{C})$.

Case II. The skew partition $\lambda / \mu$ satisfies that $\ell(\lambda)-\ell(\mu) \equiv 1(\bmod 2)$.

(1) If $o_{r} \leq e_{r}+1$, then $o_{r}^{\prime}<e_{r}^{\prime}$ and

$\kappa(\mathscr{C})=o_{s}+2 e_{s}+e_{r}+1, \quad \kappa\left(\mathscr{C}^{\prime}\right)=o_{s}+2 e_{s}-1+e_{r}^{\prime}+1=o_{s}+2 e_{s}+e_{r}+1=\kappa(\mathscr{C})$. 
(2) If $o_{r} \geq e_{r}+3$, then $o_{r}^{\prime}=o_{r}-1 \geq e_{r}+2>e_{r}^{\prime}$ and $\kappa(\mathscr{C})=o_{s}+2 e_{s}+o_{r}, \quad \kappa\left(\mathscr{C}^{\prime}\right)=o_{s}+2 e_{s}-1+o_{r}^{\prime}=o_{s}+2 e_{s}+o_{r}-2<\kappa(\mathscr{C})$.

Therefore, the inequality $\kappa\left(\mathscr{C}^{\prime}\right) \leq \kappa(\mathscr{C})$ holds under the assumption.

Lemma 5.3. If $\mathscr{C}^{\prime}=\mathscr{C}\left(\left[\begin{array}{c}(o, e) \\ (\varnothing, e)\end{array}\right] \rightarrow\left[\begin{array}{c}(\varnothing, o) \\ (o, o)\end{array}\right]\right)$, then $\kappa\left(\mathscr{C}^{\prime}\right) \leq \kappa(\mathscr{C})$.

Proof. In this case we have

$$
o_{s}^{\prime}=o_{s}+1, \quad e_{s}^{\prime}=e_{s}-1, \quad o_{r}^{\prime}=o_{r}+1, \quad e_{r}^{\prime}=e_{r}-1 .
$$

Now there are two possibilities.

Case I. The skew partition $\lambda / \mu$ satisfies that $\ell(\lambda)-\ell(\mu) \equiv 0(\bmod 2)$.

(1) If $o_{r} \leq e_{r}-2$, then $o_{r}^{\prime} \leq e_{r}^{\prime}$ and

$\kappa(\mathscr{C})=o_{s}+2 e_{s}+e_{r}, \quad \kappa\left(\mathscr{C}^{\prime}\right)=o_{s}+1+2\left(e_{s}-1\right)+e_{r}^{\prime}=o_{s}+2 e_{s}+e_{r}-2<\kappa(\mathscr{C})$.

(2) If $o_{r} \geq e_{r}$, then $o_{r}^{\prime}=o_{r}+1>e_{r}-1=e_{r}^{\prime}$ and

$$
\kappa(\mathscr{C})=o_{s}+2 e_{s}+o_{r}, \quad \kappa\left(\mathscr{C}^{\prime}\right)=o_{s}+2 e_{s}-1+o_{r}^{\prime}=o_{s}+2 e_{s}+o_{r}=\kappa(\mathscr{C}) .
$$

Case II. The skew partition $\lambda / \mu$ satisfies that $\ell(\lambda)-\ell(\mu) \equiv 1(\bmod 2)$.

(1) If $o_{r} \leq e_{r}-1$, then $o_{r}^{\prime}<e_{r}^{\prime}+1$ and

$\kappa(\mathscr{C})=o_{s}+2 e_{s}+e_{r}+1, \quad \kappa\left(\mathscr{C}^{\prime}\right)=o_{s}+2 e_{s}-1+e_{r}^{\prime}+1=o_{s}+2 e_{s}+e_{r}-1<\kappa(\mathscr{C})$.

(2) If $o_{r} \geq e_{r}+1$, then $o_{r}^{\prime}=o_{r}+1>e_{r}+1=e_{r}^{\prime}+2$ and

$$
\kappa(\mathscr{C})=o_{s}+2 e_{s}+o_{r}, \quad \kappa\left(\mathscr{C}^{\prime}\right)=o_{s}+2 e_{s}-1+o_{r}^{\prime}=o_{s}+2 e_{s}+o_{r}=\kappa(\mathscr{C}) .
$$

In both cases we have $\kappa\left(\mathscr{C}^{\prime}\right) \leq \kappa(\mathscr{C})$, as required.

Lemma 5.4. If $\mathscr{C}^{\prime}=\mathscr{C}\left(\left[\begin{array}{l}(e, e) \\ (o, e)\end{array}\right] \rightarrow\left[\begin{array}{l}(o, o) \\ (e, o)\end{array}\right]\right)$, then $\kappa\left(\mathscr{C}^{\prime}\right)<\kappa(\mathscr{C})$.

Proof. In this case, we have

$$
o_{s}^{\prime}=o_{s}+2, \quad e_{s}^{\prime}=e_{s}-2, \quad o_{r}^{\prime}=o_{r}, \quad e_{r}^{\prime}=e_{r} .
$$

Therefore,

$$
\kappa\left(\mathscr{C}^{\prime}\right)=o_{s}^{\prime}+2 e_{s}^{\prime}+\max \left(o_{r}^{\prime}, e_{r}^{\prime}+\left(\left(e_{r}^{\prime}+o_{r}^{\prime}\right) \bmod 2\right)\right)=\kappa(\mathscr{C})-2 .
$$

The desired inequality immediately follows.

Lemma 5.5. If $\mathscr{C}^{\prime}=\mathscr{C}\left(\left[\begin{array}{l}(e, o) \\ (\varnothing, e)\end{array}\right] \rightarrow\left[\begin{array}{l}(\varnothing, o) \\ (e, \varnothing)\end{array}\right]\right)$, then $\kappa\left(\mathscr{C}^{\prime}\right) \leq \kappa(\mathscr{C})$. 
Proof. Under this transformation we have

$$
o_{s}^{\prime}=o_{s}-1, \quad e_{s}^{\prime}=e_{s}, \quad o_{r}^{\prime}=o_{r}+1, \quad e_{r}^{\prime}=e_{r}-1 .
$$

Since $o_{r}+e_{r}=\ell(\lambda)-\ell(\mu)$ is invariant, there are two cases.

Case I. The skew partition $\lambda / \mu$ satisfies that $\ell(\lambda)-\ell(\mu) \equiv 0(\bmod 2)$.

(1) If $o_{r} \geq e_{r}$, then $o_{r}^{\prime} \geq e_{r}^{\prime}$ and

$$
\kappa\left(\mathscr{C}^{\prime}\right)=o_{s}^{\prime}+2 e_{s}^{\prime}+o_{r}^{\prime}=o_{s}-1+2 e_{s}+o_{r}+1=\kappa(\mathscr{C}) .
$$

(2) If $o_{r} \leq e_{r}-2$, then $o_{r}^{\prime}=o_{r}+1 \leq e_{r}-1=e_{r}^{\prime}$ and

$$
\kappa\left(\mathscr{C}^{\prime}\right)=o_{s}-1+2 e_{s}+e_{r}^{\prime}=o_{s}+2 e_{s}+e_{r}-2<\kappa(\mathscr{C}) .
$$

Case II. The skew partition $\lambda / \mu$ satisfies that $\ell(\lambda)-\ell(\mu) \equiv 1(\bmod 2)$.

(1) If $o_{r} \geq e_{r}+1$, then $o_{r}^{\prime}=o_{r}+1 \geq e_{r}+2>e_{r}^{\prime}+1$ and

$$
\kappa\left(\mathscr{C}^{\prime}\right)=o_{s}^{\prime}+2 e_{s}^{\prime}+o_{r}^{\prime}=o_{s}+2 e_{s}+o_{r}=\kappa(\mathscr{C}) .
$$

(2) If $o_{r} \leq e_{r}-1$, then $o_{r}^{\prime}=o_{r}+1 \leq e_{r}=e_{r}^{\prime}+1$ and

$$
\kappa\left(\mathscr{C}^{\prime}\right)=o_{s}^{\prime}+2 e_{s}^{\prime}+e_{r}^{\prime}+1=o_{s}+2 e_{s}+e_{r}-1<\kappa(\mathscr{C}) .
$$

Lemma 5.6. If $\mathscr{C}^{\prime}=\mathscr{C}\left(\left[\begin{array}{c}(o, o) \\ (\varnothing, o)\end{array}\right] \rightarrow\left[\begin{array}{l}(\varnothing, e) \\ (o, \varnothing)\end{array}\right]\right)$, then $\kappa\left(\mathscr{C}^{\prime}\right) \leq \kappa(\mathscr{C})$.

Proof. In this case we have

$$
o_{s}^{\prime}=o_{s}-1, \quad e_{s}^{\prime}=e_{s}, \quad o_{r}^{\prime}=o_{r}-1, \quad e_{r}^{\prime}=e_{r}+1 .
$$

There are two possibilities:

Case I. The skew partition $\lambda / \mu$ satisfies that $\ell(\lambda)-\ell(\mu) \equiv 0(\bmod 2)$.

(1) If $o_{r} \geq e_{r}+2$, then $o_{r}^{\prime}=o_{r}-1 \geq e_{r}+1=e_{r}^{\prime}$ and

$$
\kappa\left(\mathscr{C}^{\prime}\right)=o_{s}^{\prime}+2 e_{s}^{\prime}+o_{r}^{\prime}=o_{s}-1+2 e_{s}+o_{r}-1<\kappa(\mathscr{C}) .
$$

(2) If $o_{r} \leq e_{r}$, then $o_{r}^{\prime}=o_{r}-1 \leq e_{r}-1<e_{r}^{\prime}$ and

$$
\kappa\left(\mathscr{C}^{\prime}\right)=o_{s}-1+2 e_{s}+e_{r}^{\prime}=o_{s}+2 e_{s}+e_{r}=\kappa(\mathscr{C}) .
$$

Case II. The skew partition $\lambda / \mu$ satisfies that $\ell(\lambda)-\ell(\mu) \equiv 1(\bmod 2)$.

(1) If $o_{r} \geq e_{r}+3$, then $o_{r}^{\prime}=o_{r}-1 \geq e_{r}+2=e_{r}^{\prime}+1$ and

$$
\kappa\left(\mathscr{C}^{\prime}\right)=o_{s}^{\prime}+2 e_{s}^{\prime}+o_{r}^{\prime}=o_{s}+2 e_{s}+o_{r}-2<\kappa(\mathscr{C}) .
$$

(2) If $o_{r} \leq e_{r}+1$, then $o_{r}^{\prime}=o_{r}-1 \leq e_{r}<e_{r}^{\prime}+1$ and

$$
\kappa\left(\mathscr{C}^{\prime}\right)=o_{s}^{\prime}+2 e_{s}^{\prime}+e_{r}^{\prime}+1=o_{s}+2 e_{s}+e_{r}+1=\kappa(\mathscr{C}) .
$$


Therefore, in both cases we have $\kappa\left(\mathscr{C}^{\prime}\right) \leq \kappa(\mathscr{C})$.

Lemma 5.7. If

$$
\mathscr{C}^{\prime}=\mathscr{C}\left(\left[\begin{array}{l}
(e, o) \\
(o, o)
\end{array}\right] \rightarrow\left[\begin{array}{l}
(o, e) \\
(e, \varnothing)
\end{array}\right]\right) \quad \text { or } \quad \mathscr{C}^{\prime}=\mathscr{C}\left(\left[\begin{array}{l}
(o, o) \\
(e, o)
\end{array}\right] \rightarrow\left[\begin{array}{c}
(e, e) \\
(o, \varnothing)
\end{array}\right]\right),
$$

then $\kappa\left(\mathfrak{C}^{\prime}\right)=\kappa(\mathscr{C})$.

Proof. In each case we have

$$
o_{s}^{\prime}=o_{s}-2, \quad e_{s}^{\prime}=e_{s}+1, \quad o_{r}^{\prime}=o_{r}, \quad e_{r}^{\prime}=e_{r} .
$$

Therefore

$$
\kappa\left(\mathscr{C}^{\prime}\right)=o_{s}^{\prime}+2 e_{s}^{\prime}+\max \left(o_{r}^{\prime}, e_{r}^{\prime}+\left(\left(e_{r}^{\prime}+o_{r}^{\prime}\right) \bmod 2\right)\right)=\kappa(\mathscr{C}),
$$

as desired.

Lemma 5.8. If $\mathfrak{G}^{\prime}$ is one of the following possible cases

$$
\begin{aligned}
& \mathscr{C}\left(\left[\begin{array}{l}
(e, e) \\
(e, e)
\end{array}\right] \rightarrow\left[\begin{array}{l}
(e, e) \\
(e, \varnothing)
\end{array}\right]\right), \quad \mathscr{C}\left(\left[\begin{array}{l}
(e, e) \\
(o, o)
\end{array}\right] \rightarrow\left[\begin{array}{l}
(o, o) \\
(e, \varnothing)
\end{array}\right]\right), \quad \mathscr{C}\left(\left[\begin{array}{l}
(e, o) \\
(e, e)
\end{array}\right] \rightarrow\left[\begin{array}{c}
(e, o) \\
(e, \varnothing)
\end{array}\right]\right), \\
& \mathscr{C}\left(\left[\begin{array}{c}
(e, e) \\
(\varnothing, e)
\end{array}\right] \rightarrow\left[\begin{array}{l}
(\varnothing, e) \\
(e, \varnothing)
\end{array}\right]\right), \quad \mathscr{C}\left(\left[\begin{array}{l}
(o, o) \\
(o, e)
\end{array}\right] \rightarrow\left[\begin{array}{l}
(o, o) \\
(o, \varnothing)
\end{array}\right]\right), \quad \mathscr{C}\left(\left[\begin{array}{l}
(o, e) \\
(e, o)
\end{array}\right] \rightarrow\left[\begin{array}{c}
(e, o) \\
(o, \varnothing)
\end{array}\right]\right), \\
& \mathscr{C}\left(\left[\begin{array}{l}
(o, e) \\
(o, e)
\end{array}\right] \rightarrow\left[\begin{array}{l}
(o, e) \\
(o, \varnothing)
\end{array}\right]\right), \quad \mathscr{C}\left(\left[\begin{array}{c}
(o, e) \\
(\varnothing, o)
\end{array}\right] \rightarrow\left[\begin{array}{l}
(\varnothing, o) \\
(o, \varnothing)
\end{array}\right]\right),
\end{aligned}
$$

then $\kappa\left(\mathscr{C}^{\prime}\right)<\kappa(\mathscr{C})$.

Proof. In each case we have

$$
o_{s}^{\prime}=o_{s}, \quad e_{s}^{\prime}=e_{s}-1, \quad o_{r}^{\prime}=o_{r}, \quad e_{r}^{\prime}=e_{r} .
$$

Therefore

$$
\kappa\left(\mathscr{C}^{\prime}\right)=o_{s}^{\prime}+2 e_{s}^{\prime}+\max \left(o_{r}^{\prime}, e_{r}^{\prime}+\left(\left(e_{r}^{\prime}+o_{r}^{\prime}\right) \bmod 2\right)\right)<\kappa(\mathscr{C}) .
$$

Note that Lemmas 5.1-5.8 cover all possible transformations of exchanging the locations of 0's in two involved rows. Lemmas 5.2-5.4 imply that, to minimize the number of bars, we should put 0's in the skew shifted diagram such that there are as many as possible rows for which the first several squares are filled with 0's and then followed by an odd number of blank squares. Meanwhile, from Lemmas 5.5-5.8 we know that the number of rows fully filled with 0's should be as large as possible. Based on these observations, we have the following algorithm to determine the locations of 0 's for a given skew partition $\lambda / \mu$, where both $\lambda$ and $\mu$ are strict partitions. Using this algorithm we will obtain a shifted diagram with some squares filled with 0 's such that the corresponding quantity $\kappa(\mathscr{C})$ is minimized. This property allows us to determine the srank of $\lambda / \mu$. 


\section{The algorithm for determining the locations of 0 's.}

(S1) Let $\mathscr{C}_{1}=S(\lambda)$ be the initial configuration of $\lambda / \mu$ with blank squares. Set $i=1$ and $J=\{1, \ldots, \ell(\lambda)\}$.

(S2) For $i \leq \ell(\mu)$, iterate the following procedure:

(A) If $\mu_{i}=\lambda_{j}$ for some $j \in J$, then we fill the $j$-th row of $\mathscr{C}_{i}$ with 0 .

(B) If $\mu_{i} \neq \lambda_{j}$ for any $j \in J$, then there are two possibilities.

(B1) $\lambda_{j}-\mu_{i}$ is odd for some $j \in J$ and $\lambda_{j}>\mu_{i}$. Then we take the largest such $j$ and fill the leftmost $\mu_{i}$ squares with 0 in the $j$-th row of $\mathscr{C}_{i}$.

(B2) $\lambda_{j}-\mu_{i}$ is even for any $j \in J$ and $\lambda_{j}>\mu_{i}$. Then we take the largest such $j$ and fill the leftmost $\mu_{i}$ squares by 0 in the $j$-th row of $\mathscr{C}_{i}$.

Denote the new configuration by $\mathscr{C}_{i+1}$. Set $J=J \backslash\{j\}$.

(S3) Set $\mathscr{b}^{*}=\mathscr{C}_{i}$, and we get the desired configuration.

We emphasize that although the above algorithm does not necessarily generate a bar tableau, it is sufficient for the computation of the srank of a skew partition.

Using the arguments in the proofs of Lemmas 5.1-5.8, we can derive the following crucial property of the configuration $\mathscr{C}^{*}$.

Proposition 5.9. For any configuration $\mathscr{b}$ of 0 's in the skew shifted diagram of $\lambda / \mu$, we have $\kappa\left(\mathscr{C}^{*}\right) \leq \kappa(\mathscr{C})$.

Proof. Given any configuration $\mathscr{b}$, we shall prove that $\mathscr{C}^{*}$ can be obtained from $\mathscr{C}$ through a sequence of transformations by exchanging the locations of 0 's in two certain rows without increasing the quantity $\kappa(\mathscr{C})$.

Suppose that, for some $i, j, k$, the $j$-th row of $\mathscr{C}$ contains $m(m \geq 0)$ squares labeled 0 , the $k$-th row contains $\mu_{i}$ squares labeled 0 , and $\mu_{i}=\lambda_{j}$. Let $\mathscr{C}^{\prime}$ be the configuration obtained from $\mathscr{C}$ by exchanging the locations of 0 's in these two rows. Note that this transformation falls into those cases of Lemmas 5.5-5.8. Thus $\kappa\left(\mathscr{C}^{\prime}\right) \leq \kappa(\mathscr{C})$. Continue in this way until there are no rows which can be fully filled with 0 's. For convenience, we still denote the resulting configuration by $\mathscr{C}$.

Then suppose that $i$ is the least number such that there does not exist $j$ satisfying $\mu_{i}=\lambda_{j}$ and the $k$-th row contains exactly $\mu_{i}$ squares filled with 0 's. If no such $i$ exists, then $\mathscr{C}$ is already $\mathscr{C}^{*}$. If there exists some $j$ such that $\lambda_{j}>\mu_{i}$ and $\lambda_{j}-\mu_{i}$ is odd, then find the largest such $j$ and exchange the locations of 0's in the $k$-th row and the $j$-th row. Otherwise, find the largest number $j$ such that $\lambda_{j}>\mu_{i}$, then we also exchange the locations of 0 's in the $k$-th row and the $j$-th row. It is straightforward to verify that these two kinds of transformations will fall into the cases of Lemmas 5.2-5.4. Let $\mathscr{C}^{\prime}$ be the configuration obtained from $\mathscr{C}$ by exchanging the locations of the 0's in the two rows involved. Then $\kappa\left(\mathscr{C}^{\prime}\right) \leq \kappa(\mathscr{C})$. Continuing in this way, the resulting configuration will become $\mathscr{C}^{*}$ according to the algorithm. At each step the index $j$ should be chosen from those indices which 
are not used in the previous steps, as described in the algorithm. Therefore, we have $\kappa\left(\mathscr{C}^{*}\right) \leq \kappa(\mathscr{C})$.

Theorem 5.10. Given a skew partition $\lambda / \mu$, let $\mathscr{C}^{*}$ be the configuration of 0 's obtained by applying the algorithm described above. Then

$$
\operatorname{srank}(\lambda / \mu)=\kappa\left(\mathfrak{C}^{*}\right) .
$$

Proof. Suppose that for the configuration $\mathscr{C}^{*}$ there are $o_{r}^{*}$ rows of odd size with blank squares, and there are $o_{s}^{*}$ rows with at least one square filled with 0 and an odd number of blank squares. Likewise we let $e_{r}^{*}$ and $e_{s}^{*}$ denote the number of remaining rows. By definition,

$$
\kappa\left(\mathfrak{C}^{*}\right)=o_{s}^{*}+2 e_{s}^{*}+\max \left(o_{r}^{*}, e_{r}^{*}+\left(\left(e_{r}^{*}+o_{r}^{*}\right) \bmod 2\right)\right) .
$$

By Proposition 2.3, for each configuration $\mathscr{b}$ the number of bars in a minimal bar tableau is greater than or equal to $\kappa(\mathscr{C})$, and thus it is greater than or equal to $\kappa\left(\mathscr{C}^{*}\right)$ due to Proposition 5.9. It suffices to confirm the existence of a skew bar tableau, say $T$, with $\kappa\left(\mathscr{C}^{*}\right)$ bars.

Note that it is possible that the configuration $\mathscr{C}^{*}$ is not admissible. The key idea of our proof is to move 0's in the diagram such that the resulting configuration $\mathscr{C}^{\prime}$ is admissible and $\kappa\left(\mathfrak{C}^{\prime}\right)=\kappa\left(\mathfrak{C}^{*}\right)$. To achieve this goal, we will use the numbers $\left\{1,2, \ldots, \kappa\left(\mathfrak{C}^{*}\right)\right\}$ to fill up the blank squares of $\mathscr{C}^{*}$ guided by the rule that the bars of Type 2 or Type 3 will occur before bars of Type 1 .

Let us consider the rows without 0's, and there are two possibilities:
(A) $o_{r}^{*} \geq e_{r}^{*}$,
(B) $o_{r}^{*}<e_{r}^{*}$.

In Case (A) we choose a row of even size and a row of odd size, and fill up these two rows with $\kappa\left(\mathscr{C}^{*}\right)$ to generate a bar of Type 3. Then we continue to choose a row of even size and a row of odd size, and fill up these two rows with $\kappa\left(\bullet^{*}\right)-1$. Repeat this procedure until all even rows are filled up. Finally, we fill the remaining rows of odd size with $\kappa\left(\mathfrak{C}^{*}\right)-e_{r}^{*}, \kappa\left(\mathfrak{C}^{*}\right)-e_{r}^{*}-1, \ldots, \kappa\left(\mathfrak{C}^{*}\right)-o_{r}^{*}+1$ to generate bars of Type 2 .

In Case (B) we choose the row with the $i$-th smallest even size and the row with the $i$-th smallest odd size and fill their squares with the number $\kappa\left(\mathscr{C}^{*}\right)-i+1$ for $i=1, \ldots, o_{r}^{*}$. In this way, we obtain $o_{r}^{*}$ bars of Type 3 . Now consider the remaining rows of even size without 0 's. There are two subcases.

(B1) The remaining diagram, obtained by removing the previous $o_{r}^{*}$ bars of Type 3, does not contain any row with only one square. Under this assumption, it is possible to fill the squares of a row of even size with the number $\kappa\left(\mathscr{C}^{*}\right)-o_{r}^{*}$ except the leftmost square. This operation will result in a bar of Type 1. After 
removing this bar from the diagram, we may combine this leftmost square of the current row and another row of even size, if it exists, to generate a bar of Type 3. Repeating this procedure until there are no more rows of even size, we obtain a sequence of bars of Type 1 and Type 3. Evidently, if $e_{r}^{*}-o_{r}^{*}$ is odd, there is a bar of Type 2 with only one square. Thus we have $\max \left(o_{r}^{*}, e_{r}^{*}+\left(\left(e_{r}^{*}+o_{r}^{*}\right) \bmod 2\right)\right)$ bars.

(B2) The remaining diagram, obtained by removing the previous $o_{r}^{*}$ bars of Type 3 , contains a row composed of the unique square filled with 0 . In this case, we will move this 0 into the leftmost square of a row of even size; see Figure 2. Denote this new configuration by $\mathscr{C}^{\prime}$; from Lemma 5.6 we see that $\kappa\left(\mathfrak{C}^{*}\right)=$ $\kappa\left(\mathscr{C}^{\prime}\right)$. If we start with $\mathscr{C}^{\prime}$ instead of $\mathscr{C}^{*}$, by a similar construction, we get $\max \left(o_{r}^{\prime}, e_{r}^{\prime}+\left(\left(e_{r}^{\prime}+o_{r}^{\prime}\right) \bmod 2\right)\right)$ bars, occupying the rows without 0 's in the diagram.

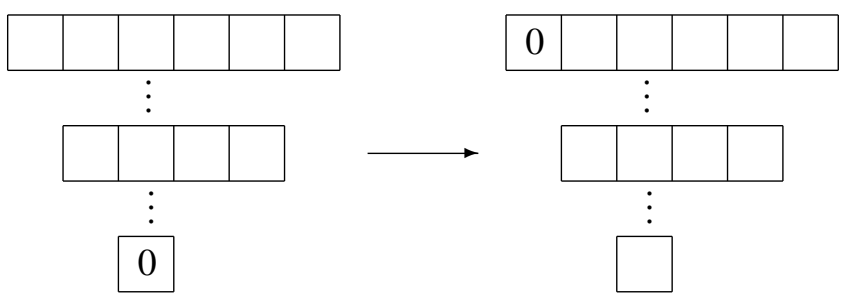

Figure 2. Vacating the unique square at the bottom of the diagram.

Without loss of generality, we may assume that for the configuration $\mathscr{C}^{*}$ the rows without 0 's in the diagram have been occupied by the bars with the first $\max \left(o_{r}^{*}, e_{r}^{*}+\left(\left(e_{r}^{*}+o_{r}^{*}\right) \bmod 2\right)\right)$ largest positive integers of $\left\{1,2, \ldots, \kappa\left(\mathscr{C}^{*}\right)\right\}$. By removing these bars and reordering the remaining rows, we may get a shifted diagram with which we can continue the above procedure to construct a bar tableau.

At this point, it is necessary to show that it is possible to use $o_{s}^{*}+2 e_{s}^{*}$ bars to fill this diagram. In doing so, we process the rows from bottom to top. If the bottom row is completely filled with 0 's, then we continue to deal with the row above the bottom row. If the bottom row has an odd number of blank squares, then we simply assign the symbol $o_{s}^{*}+2 e_{s}^{*}$ to these squares to produce a bar of Type 1. Otherwise, we fill the rightmost square of the bottom row with $o_{s}^{*}+2 e_{s}^{*}$ and the remaining squares with $o_{s}^{*}+2 e_{s}^{*}-1$. Suppose that we have filled $i$ rows from the bottom and all the involved bars have been removed from the diagram. Then we consider the $(i+1)$-st row from the bottom. Let $t$ denote the largest number not greater than $o_{s}^{*}+2 e_{s}^{*}$ which has not been used before. If all squares in the $(i+1)$-st row are filled with 0 's, then we continue to deal with the $(i+2)$-nd row. If the number of blank squares in the $(i+1)$-st row is odd, then we fill these squares with $t$. If the 
number of blank squares in the $(i+1)$-st row is even, then we are left with two cases:

$\left(\mathrm{A}^{\prime}\right)$ The rows of the diagram obtained by removing the rightmost square of the $(i+1)$-st row have distinct lengths. In this case, we fill the rightmost square with $t$ and the remaining blank squares of the $(i+1)$-st row with $t-1$.

( $\left.\mathrm{B}^{\prime}\right)$ The removal of the rightmost square of the $(i+1)$-st row does not result in a bar tableau. Suppose that the $(i+1)$-st row has $m$ squares in total. It can only happen that the row underneath the $(i+1)$-st row has $m-1$ squares and all these squares are filled with 0's. By interchanging the locations of the 0's in these two rows, we get a new configuration $\mathscr{C}^{\prime}$; see Figure 3. From Lemma 5.7 we deduce that $\kappa\left(\mathscr{C}^{*}\right)=\kappa\left(\mathscr{C}^{\prime}\right)$. So we can transform $\mathscr{C}^{*}$ to $\mathscr{C}^{\prime}$ and continue to fill up the $(i+1)$-st row.

\begin{tabular}{|l|l|l|l|l|l|l}
\hline 0 & 0 & 0 & & & & \\
\hline & 0 & 0 & 0 & 0 & 0 & 0
\end{tabular}$\longrightarrow$\begin{tabular}{|l|l|l|l|l|l|l|}
\hline 0 & 0 & 0 & 0 & 0 & 0 & \\
\hline & 0 & 0 & 0 & & & \\
\hline
\end{tabular}

Figure 3. Interchanging the locations of the 0's in two neighboring rows.

Finally, we arrive at a shifted diagram whose rows are all filled up. Clearly, for rows containing at least one 0 there are $o_{s}^{*}+2 e_{s}^{*}$ bars that are generated in the construction. For rows containing no 0's there are $\max \left(o_{r}^{*}, e_{r}^{*}+\left(\left(e_{r}^{*}+o_{r}^{*}\right) \bmod 2\right)\right)$ bars that are generated. It has been shown that during the procedure of filling the diagram with nonnegative numbers if the configuration $\mathscr{C}^{*}$ is transformed to another configuration $\mathscr{C}^{\prime}$, then $\kappa\left(\mathscr{C}^{\prime}\right)$ remains equal to $\kappa\left(\mathscr{C}^{*}\right)$. Hence the above procedure leads to a skew bar tableau of shape $\lambda / \mu$ with $\kappa\left(\mathscr{C}^{*}\right)$ bars.

\section{Acknowledgments}

This work was supported by the 973 Project, the PCSIRT Project of the Ministry of Education, and the National Science Foundation of China.

\section{References}

[Clifford 2003] P. Clifford, Algebraic and combinatorial properties of minimal border strip tableaux, Ph.D. thesis, Massachusetts Institute of Technology, 2003.

[Clifford 2005] P. Clifford, "Minimal bar tableaux", Ann. Combin. 9:3 (2005), 281-291. MR 2006j: 05211 Zbl 1073.05072

[Clifford and Stanley 2004] P. Clifford and R. P. Stanley, "Bottom Schur functions", Electron. J. Combin. 11:1 (2004), Research Paper 67, 16 pp. MR 2005f:05167 Zbl 1061.05097

[Hamel 1996] A. M. Hamel, "Pfaffians and determinants for Schur $Q$-functions", J. Combin. Theory Ser. A 75:2 (1996), 328-340. MR 98c:05161 Zbl 0858.05098 
[Hoffman and Humphreys 1992] P. N. Hoffman and J. F. Humphreys, Projective representations of the symmetric groups. Q-functions and shifted tableaux, Oxford Univ. Press, New York, 1992. MR 94f:20027 Zbl 0777.20005

[Humphreys 1986] J. F. Humphreys, "Blocks of projective representations of the symmetric groups", J. London Math. Soc. (2) 33:3 (1986), 441-452. MR 87k:20027 Zbl 0633.20007

[Józefiak 1989] T. Józefiak, "Characters of projective representations of symmetric groups", Exposition. Math. 7:3 (1989), 193-247. MR 90f:20018 Zbl 0693.20009

[Józefiak and Pragacz 1991] T. Józefiak and P. Pragacz, "A determinantal formula for skew Qfunctions", J. London Math. Soc. (2) 43:1 (1991), 76-90. MR 92d:05175 Zbl 0761.20007

[Macdonald 1995] I. G. Macdonald, Symmetric functions and Hall polynomials, 2nd ed., Oxford Univ. Press, New York, 1995. MR 96h:05207 Zbl 0824.05059

[Morris 1962] A. O. Morris, "The spin representation of the symmetric group", Proc. London Math. Soc. (3) 12 (1962), 55-76. MR 25 \#133 Zbl 0104.25202

[Morris 1965] A. O. Morris, "The spin representation of the symmetric group", Canad. J. Math. 17 (1965), 543-549. MR 31 \#240 Zbl 0135.05602

[Morris 1979] A. O. Morris, "The projective characters of the symmetric group-an alternative proof”, J. London Math. Soc. (2) 19:1 (1979), 57-58. MR 58 \#22262 Zbl 0393.20010

[Nazarov 1988] M. L. Nazarov, "An orthogonal basis in irreducible projective representations of the symmetric group", Funktsional. Anal. i Prilozhen. 22:1 (1988), 77-78. MR 89f:20019 Zbl 0658. 20010

[Nazarov and Tarasov 2002] M. Nazarov and V. Tarasov, "On irreducibility of tensor products of Yangian modules associated with skew Young diagrams”, Duke Math. J. 112:2 (2002), 343-378. MR 2003b:17021 Zbl 1027.17013

[Sagan 1987] B. E. Sagan, "Shifted tableaux, Schur $Q$-functions, and a conjecture of R. Stanley", J. Combin. Theory Ser. A 45:1 (1987), 62-103. MR 88f:05011 Zbl 0661.05010

[Schur 1911] I. Schur, "Über die Darstellung der symmetrischen und der alternierenden Gruppe durch gebrochene lineare Substitutionen”, J. Reine Angew. Math. 139 (1911), 155-250. Zbl 42. 0154.02

[Stanley 2002] R. P. Stanley, "The rank and minimal border strip decompositions of a skew partition”, J. Combin. Theory Ser. A 100:2 (2002), 349-375. MR 2003m:05210 Zbl 1018.05105

[Stembridge 1989] J. R. Stembridge, "Shifted tableaux and the projective representations of symmetric groups”, Adv. Math. 74:1 (1989), 87-134. MR 90k:20026 Zbl 0677.20012

[Stembridge 1990a] J. R. Stembridge, "Nonintersecting paths, Pfaffians, and plane partitions", Adv. Math. 83:1 (1990), 96-131. MR 91h:05014 Zbl 0790.05007

[Stembridge 1990b] J. R. Stembridge, "On symmetric functions and the spin characters of $S_{n}$ ", pp. 433-453 in Topics in algebra, Part 2 (Warsaw, 1988), edited by S. Balcerzyk et al., Banach Center Publ. 26, PWN, Warsaw, 1990. MR 93e:20018 Zbl 0774.20011

[Stembridge] J. R. Stembridge, “The SF package”, Available at http://www.math.lsa.umich.edu/ $\sim$ jrs/maple.html\#SF.

[Worley 1984] D. Worley, A theory of shifted Young tableaux, Ph.D. thesis, Massachusetts Institute of Technology, 1984.

Received March 9, 2009. 
William Y. C. Chen

Center for Combinatorics, LPMC-TJKLC

NANKAI UNIVERSITY

NO. 94 WEIJIN ROAD

NANKAI DISTRICT

TIANJIN 300071

CHINA

chen@nankai.edu.cn

http://www.billchen.org/

DONNA Q. J. DOU

CENTER FOr COMBINATORICS, LPMC-TJKLC NANKAI UNIVERSITY

NO. 94 WEIJIN ROAD

NANKAI DISTRICT

TIANJIN 300071

CHINA

qjdou@cfc.nankai.edu.cn

ROBERT L. TANG

Center for Combinatorics, LPMC-TJKLC NANKAI UNIVERSITY

NO. 94 WEIJIN ROAD

NANKAI DISTRICT

TIANJIN 300071

CHINA

tangling@cfc.nankai.edu.cn

ARTHUR L. B. YANG

CENTER FOR COMBINATORICS, LPMC-TJKLC NANKAI UNIVERSITY

NO. 94 WEIJIN ROAD

NANKAI DISTRICT

TIANJIN 300071

CHINA

yang@nankai.edu.cn

http://www.combinatorics.net.cn/homepage/yang/ 